\title{
Genetic alterations in cell cycle regulation-associated genes may promote primary progression of gastrointestinal stromal tumors
}

\author{
Peifeng $\mathrm{Li}^{1,2} \cdot$ Mingyang $\mathrm{Li}^{1} \cdot \mathrm{Kaijing} \mathrm{Wang}^{1} \cdot$ Yixiong $\mathrm{Liu}^{1} \cdot$ Yingmei Wang ${ }^{1} \cdot$ Danhui Zhao ${ }^{1} \cdot \mathrm{Jia}^{\mathrm{Chai}}{ }^{1} \cdot \mathrm{Jing} \mathrm{Ma}^{1} \cdot$ \\ Xia $\mathrm{Li}^{1} \cdot$ Jie Wei ${ }^{1} \cdot$ Linni Fan $^{1} \cdot$ Feng Zhang ${ }^{1} \cdot$ Jing Ye ${ }^{1} \cdot$ Qingguo Yan ${ }^{1} \cdot$ Shuangping Guo ${ }^{1} \cdot$ Zhe Wang $^{1}$
}

Received: 19 May 2019 / Accepted: 21 August 2019 / Published online: 30 September 2019

(c) The Author(s), under exclusive licence to United States and Canadian Academy of Pathology 2019

\begin{abstract}
Gastrointestinal stromal tumors (GISTs) are one of the most common mesenchymal tumor types and usually contain KIT or PDGFRA mutations. GISTs with concomitant low- and high-grade components are seen in clinical practice. Herein, we retrospectively analyzed the histological characteristics and immunohistochemical results of 22 GIST cases with concomitant low- and high-grade tumors. Whole-exome sequencing (WES) was performed on ten pairs of high-grade GIST specimens and matched low-grade samples. Differential oncogenes mutated only in high-grade GISTs were identified, which were confirmed by Sanger sequencing. Fluorescence in situ hybridization was employed to detect MYC copy number variation. High-grade GISTs were more likely to have lower CD34 expression and a higher Ki-67 proliferation index compared to the matched low-grade tumors. WES identified 30 differential cancer-associated genes mutated only in highgrade GISTs; Sanger sequencing confirmed ten relevant differential oncogenic mutations in nine genes (MGA, ARIDIA, LATS2, MAX, PIK3CA, RB1, RPS6KB2, SDHA, and SETD2). Two patients had MGA mutations, whereas other gene mutations occurred in only one patient. Most of the differential cancer-associated genes are mainly involved in cell cycle control. MYC copy number gain was a common genetic variation. High-grade GISTs revealed more MYC copy number gains than matched low-grade tumors, and low-grade GISTs with coexisting high-grade components showed more MYC copy number gains than pure low-grade GISTs. Moreover, MYC copy number gain was positively correlated with the mitotic index and Ki-67 proliferation index. Alterations in cell cycle regulation-associated genes, such as genetic mutations and MYC copy number gain, may promote primary progression from low-grade GISTs to high-grade tumors by regulating tumor cell proliferation.
\end{abstract}

\section{Introduction}

Gastrointestinal stromal tumors (GISTs), which are the most common mesenchymal tumors in the digestive tract, span a biological behavior spectrum that ranges from benign to

Supplementary information The online version of this article (https:// doi.org/10.1038/s41374-019-0322-x) contains supplementary material, which is available to authorized users.

Zhe Wang

zhwang@fmmu.edu.cn

1 State Key Laboratory of Cancer Biology, Department of Pathology, Xijing Hospital and School of Basic Medicine, The Fourth Military Medical University, Xi' an, Shaan Xi, China

2 Department of Pathology, The 960th Hospital of PLA, Jinan, Shandong, China malignant. Activating mutations in the KIT or PDGFRA (platelet-derived growth factor A) genes are frequently identified in most GISTs. It is estimated that these mutations result in structural changes to tyrosine kinase receptors and ligandindependent constitutive autoactivation of kinases, which function in signal transduction pathways that promote proliferation and inhibit apoptosis; this in turn results in GIST tumorigenesis [1]. In addition, most KIT/PDGFRA wild-type GISTs usually have loss of function of succinate dehydrogenase (SDH) or mutations in the BRAF/KRAS or NFI genes [2,3]. With advances in comprehensive genomic profiling, many novel molecular events have been described in GISTs that lack or feature KIT/PDGFRA mutations, such as mutations in PIK3CA, MEN1, MAX, and ETV6-NTRK3 fusion [4-6]. Some mutations are related to the progression of GIST toward a tumor with more aggressive biological behavior. PIK3CA mutations have been detected in large or metastatic KIT-mutant GISTs, which suggest that PIK3CA-mutant clones 
have a proliferative advantage during disease progression [4]. SETD2 mutations have been identified only in high-risk/ metastatic GISTs, and assessing SETD2 genetic status has been recommended to guide risk stratification [7]. However, most of these studies of large sample sizes were based on individual cases of either low- or high-risk GIST, and thus a wide comprehensive genomic study of paired samples of different GIST grades from the same cases is lacking.

In this study, for the first time, we identified differential mutations in a series of GISTs with concomitant low- and high-grade tumors via whole-exome sequencing (WES) and Sanger sequencing and analyzed the potential driver molecular abnormalities. Nine differential cancer-associated genes that were mutated only in high-grade GISTs were identified, most of which are mainly involved in cell cycle control. Mutations in MGA and MAX were detected in three highgrade GISTs, which suggest that the MGA/MAX/MYC network plays a role in tumor progression. Furthermore, we determined the $M Y C$ gene status using fluorescence in situ hybridization (FISH). Mutations in cell cycle regulationassociated genes and $M Y C$ copy number gain may promote cell proliferation and result in the progression of GISTs.

\section{Materials and methods}

\section{Patients and tumor samples}

We re-reviewed the histological characteristics and immunohistochemical results of 1183 GIST cases from January 2012 to June 2018 in the archived pathology database of Xijing Hospital (Xi' an, China) and identified 22 GIST cases with concomitant low- and high-grade components. In addition, $M Y C$ gene status was determined in 86 cases of pure low- or high-grade GIST. By definition, the low-grade component contains a population of spindle cells without marked cellular atypia and hypercellularity and $\leq 5$ mitoses per 50 high-power fields (HPF) (a total area of $5 \mathrm{~mm}^{2}$ ). In contrast, the high-grade component consists of spindle or epithelioid cells with obvious pleomorphism or atypia and hypercellularity and with $>5$ mitoses per $50 \mathrm{HPF}$. GISTs with concomitant low- and high-grade tumors contain two morphologically different neoplastic components, which either gradually or abruptly transition to one another; these components also show dramatic differences in mitotic activity. To distinguish these two coexisting components more strictly, the mitotic index of the high-grade tumor was found to exceed that of the low-grade tumor by $\geq 5$ mitoses per $5 \mathrm{~mm}^{2}$. Written informed consent was obtained from each participant, and the study was approved by the Xijing Hospital institutional ethical committee. This study was performed in accordance with the principles embodied in the Declaration of Helsinki.

\section{Whole-exome sequencing}

We selected 22 clinical cases of GIST with concomitant low- and high-grade components, and of these, ten pairs of high-grade tumor specimens and matched low-grade samples had sufficiently high DNA quality for WES. Hematoxylin-eosin (HE)-stained tumor tissue sections were used as a guide for localizing the high- or low-grade tumors. The targeted neoplastic tissues containing a minimum of $85 \%$ tumor nuclei were manually microdissected from unstained, $10-\mu \mathrm{m}$-thick formalin-fixed, paraffin-embedded (FFPE) sections. All 20 GIST specimens were obtained from primary tumors, and the patients from which they were derived had not undergone radiation, chemotherapy, or targeted therapy with tyrosine kinase inhibitors prior to surgery. Genomic DNA was extracted using a QIAamp DNA FFPE Tissue Kit (Qiagen Inc., Hilden, Germany) according to the manufacturer's recommendations. Hybridization-captured, adapter ligation-based libraries were synthesized using an Agilent SureSelect Human All Exon Kit (Santa Clara, CA, USA), which was designed to enrich 334,378 targeted exonic regions of 20,965 genes. The WES libraries were then amplified and quality-checked, and Illumina HiSeq Platform sequencing (Novogene Bioinformatics Technology Co. Ltd, Beijing, China) was performed. The average sequencing depth of the target regions was $>100 \times$.

\section{Genomic analysis}

After raw data generation by base calling, the paired-end reads were trimmed with AdapterRemoval (http://ada pterremoval.readthedocs.io/) to remove stretches of lowquality bases $(<\mathrm{Q} 10)$ and adapters in the sequences. The paired-end reads were then aligned on the human reference genome GRCh37 (hg19) using the Burrows-Wheeler Aligner (http://bio-bwa.sourceforge.net/) and Samblaster (https:// github.com/GregoryFaust/samblaster). Variant calling was performed using the Genome Analysis Toolkit (https://softwa re.broadinstitute.org/gatk/) and SAMtools (http://www.htslib. org/). To maximize sensitivity in heterogeneous GIST specimens, the test was validated to detect base substitutions and short insertions and deletions (indels) with a threshold depth of coverage $>5 \times$ and a variant allele frequency $\geq 10 \%$. Furthermore, point mutations and small indels in genes were annotated for their functional effect on their respective proteins via ANNOVAR (Version 2013Aug23) (http://www. openbioinformatics.org/annovar/). Nonsynonymous and nonsense single-nucleotide variations (SNVs), frameshift/nonframeshift indels, and splice region mutations were selected. Variants present in the Exome Aggregation Consortium (http://exac.broadinstitute.org/) or 1000 Genomes databases (https://www.1000genomes.org/) with a frequency $>1 \%$, 
which indicated potential germline variants, were excluded from the analysis. All indels and SNVs that were predicted to be deleterious by at least one of four prediction tools (SIFT, PolyPhen-2_HDIV, MutationTaster, CADD) were included in further analyzes. Potential candidate drivers were determined according to the Catalog of Somatic Mutations in Cancer (COSMIC, http://cancer.sanger.ac.uk/census), MDG125 [8], SMG127 [9], CDG291 [10], and published references [11]. Similar and differential mutations in cancerassociated genes were obtained by Venn diagram analyses of high- and low-grade tumors. To identify the over-represented pathways, gene set enrichment analysis was performed using web-based OmicShare KEGG (Kyoto Encyclopedia of Genes and Genomes) Enrichment Analysis (http://www.omicshare. com/tools/), and related references were reviewed for mutated genes not included in the data.

\section{Sanger sequencing}

To validate the relevant differential oncogenic mutations detected only in high-grade GISTs by WES, PCR (polymerase chain reaction) primers were designed according to the mutated sites using Primer Premier 5.0 software. DNA fragments flanking the putative mutations were amplified over 39 cycles in a $50 \mu$ reaction mixture, which contained $45 \mu \mathrm{l}$ Golden Star T6 Super PCR Mix (Tsingke, Xi'an, China), $4 \mu \mathrm{l}$ forward and reverse primers, and $1 \mu$ lemplate genomic DNA per reaction. The PCR products were purified with a Tsingke PCR Purification Kit and were sequenced for low- and high-grade tumors with a Big Dye Terminator v1.1 Cycle Sequencing Kit (Applied Biosystems, Monza, Italy) in an ABI 3730xl Genetic Analyzer (Applied Biosystems). To improve sequencing accuracy and to exclude analytical errors, all sequencing analyses were carried out on both strands and were repeated on the PCR products. The alignments of the high- and lowgrade tumor sequencing data and the reference sequence were compared using SnapGene (version 2.3.2).

\section{Fluorescence in situ hybridization}

To verify the status of $M Y C$ copy number variation (CNV), FISH was performed on GIST samples using a MYC (8q24) probe (Ambiping Pharmaceutical Technology Co. Ltd., Guangzhou, China) according to the manufacturer's instructions. Copy number gain was determined above a $15 \%$ threshold with more than two red signals per nuclei.

\section{Statistical analysis}

The Wilcoxon signed rank test was used to compare the MYC copy number gain and immunohistochemical expression between high-grade and low-grade tumors in cases with both components. The Mann-Whitney U test and the Spearman rank correlation test were used to analyze the relationship between $M Y C$ copy number gain and tumor features in pure low- or high-grade GIST.

\section{Results}

\section{Clinicopathological features of GISTs with concomitant low- and high-grade components}

Seventeen $(77.3 \%)$ of the 22 patients were male. The age at the time of diagnosis ranged from 37 to 77 years, and the average age was $54.9 \pm 9.4$ years (median, 56 years). The age distribution was similar to that of general GISTs. The tumor was located in the stomach in five cases, in the small intestine in 16 cases, and in the colon in one case. All patients had a maximum tumor diameter $\geq 4 \mathrm{~cm}$ (range, $4-18 \mathrm{~cm}$ ), and nine cases $(40.91 \%)$ had multiple nodules. Single or multiple metastases were found during surgery in six patients. The proportion of high-grade components in whole tumors of each patient was $10-85 \%$. The risk stratification of the tumors was assigned based on location, size, and mitotic index in the high-grade component; therefore, all GISTs were high-risk or very high-risk tumors (Table 1).

Microscopically, two tumor components with different cell densities and cell morphologies were present in the same nodule or in different nodules (Fig. 1a). Low-grade GISTs usually exhibited a predominantly spindle cell morphology, a lower cell density and distinct extracellular collagen fibers with lower mitotic activity (mitotic counts were 1-2 per $50 \mathrm{HPF}$ ) (Fig. 1b). High-grade GISTs frequently showed rhabdoid to epithelioid histology and a significantly higher cell density that revealed sarcomatoid features with significant nuclear atypia and pleomorphism, and even multinucleation (Fig. 1c, d). Small focal or massive necrosis was present in 17 cases of high-grade GIST (Fig. 1e). The mitotic index in the high-grade tumors was 6-90 per $50 \mathrm{HPF}$, which was significantly increased compared with the low-grade components $(P=0.000)$ (Table 1).

Most of low- and high-grade GISTs demonstrated diffuse and strong immunohistochemical reactivity for CD117 and DOG1, and there was no significant difference in expression between the two tumor components (Fig. S1). Compared with the matched low-grade GISTs, 14 patients had lower CD34 expression in high-grade tumors $(P=0.000)$, and of these, five cases showed diffuse strong positivity in lowgrade tumors and negativity in high-grade tumors (Fig. 2a, b). Eight patients had similar CD34 expression in the lowand high-grade components, among which five cases were diffusely positive and one case was negative. The high- and low-grade tumors had significantly different Ki-67 


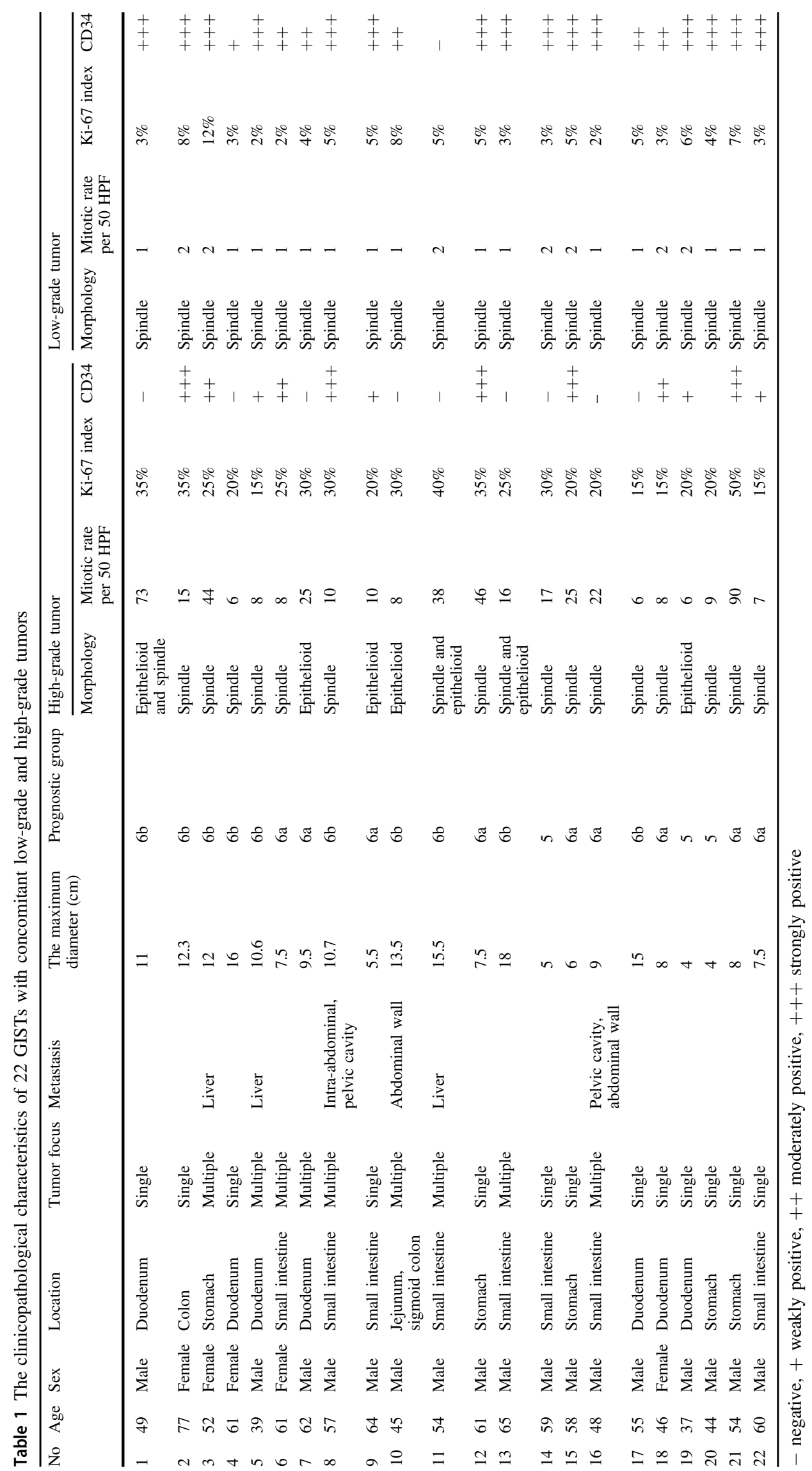



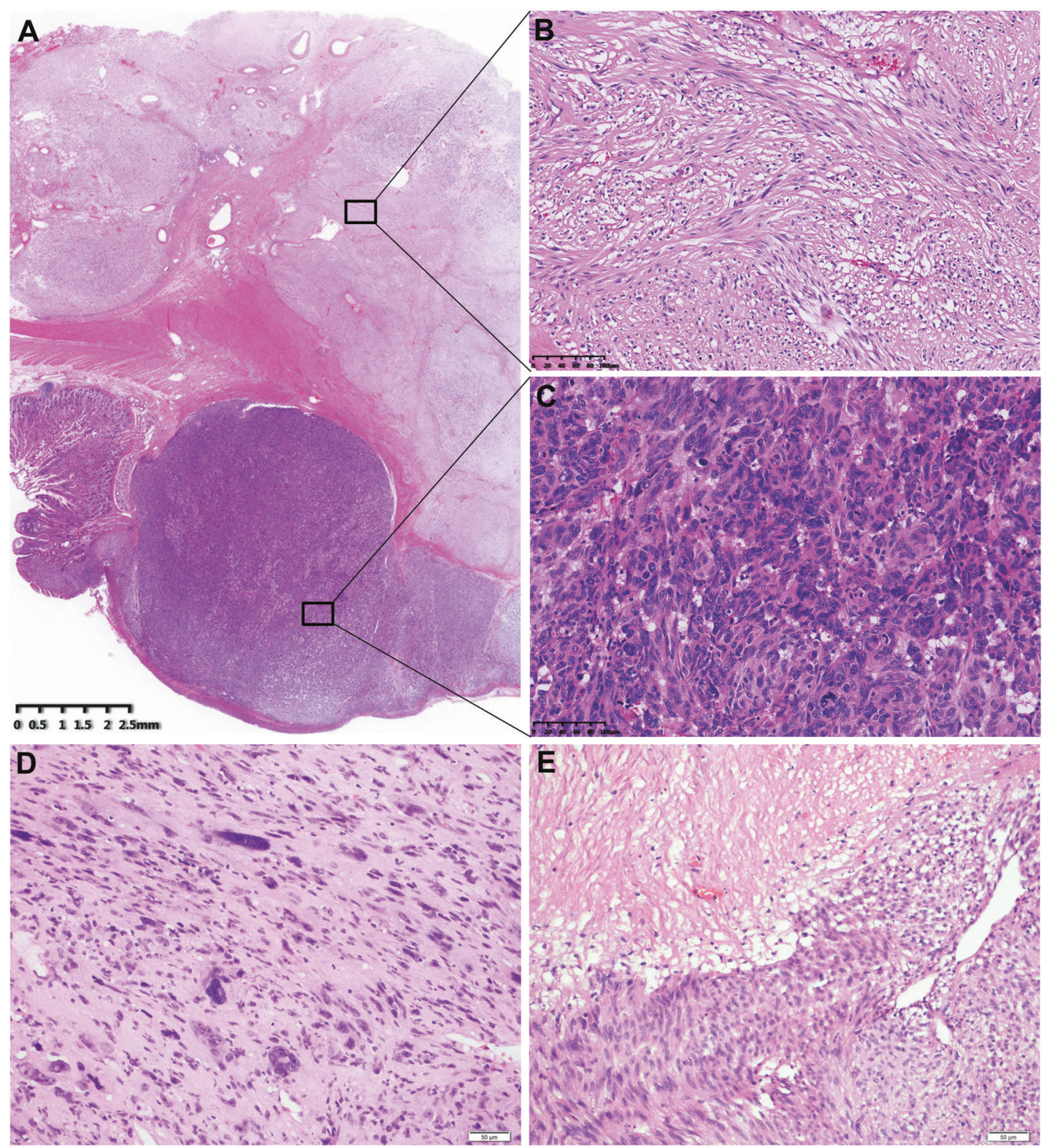

Fig. 1 Pathological characteristics of GISTs with coexisting low- and high-grade components. a Two tumor components with different cell densities were noticeably separated by smooth muscle tissue (hematoxylin-eosin staining $(\mathrm{HE}), \times 6)$. b The low-grade component displayed bland spindle cell histology, a lower cell density and rare

mitotic figures (HE, $\times 200$ ). c The high-grade tumor contained short spindle cells with obvious atypia, a brisk mitotic rate and a higher cell density (HE, $\times 200$ ). d The high-grade GIST exhibited obvious pleomorphism with tumor giant cells and multinuclear cells $(\mathrm{HE}, \times 200)$. e Necrosis was often observed in high-grade tumors (HE, $\times 200$ )

proliferation indexes $(P=0.000)$. The Ki-67 index of highgrade GISTs ranged from $15-50 \%$, while that of low-grade tumors ranged from 2 to $8 \%$ (Fig. 2c, d, Table 1).

\section{High-grade GISTs contain differential mutations in cell cycle-related genes}

WES was performed on ten cases of high-grade GIST and on the corresponding low-grade tumors. An average 69.5 million reads per sample were obtained, which produced an average coverage depth of $71 \times-160 \times$ on target regions per sample. We analyzed candidate oncogenes with potentially deleterious mutations in the coding sequence and splicing regions, and 802 mutations in 247 oncogenes or tumor suppressor genes were detected (Fig. 3). The number of variants per specimen was 27-52, with a median of 37 . Both high-grade and low-grade GISTs shared some of the mutated genes (193/247, 78.14\%), and only a few differential oncogenes were mutated only in high-grade tumors $(12.15 \%)$ or low-grade tumors $(9.71 \%)$. The KIT gene, which was mutated in all 20 samples, showed the same mutation pattern in the two components from each patient; 


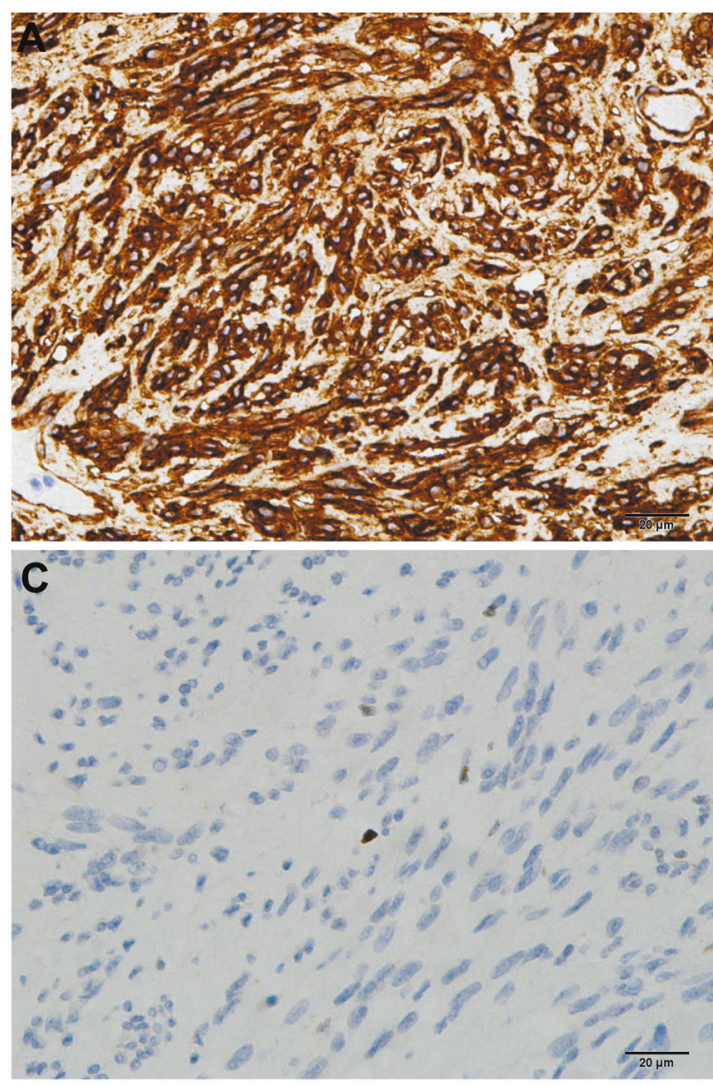

Fig. 2 Immunohistochemical features of GISTs with coexisting lowand high-grade tumor components. a The low-grade tumors showed diffuse immunohistochemical positivity for CD34 (immunohistochemical staining (IHC), ×400); b Matched high-grade GISTs were

this finding suggested that high-grade and low-grade tumors in the same patient might arise from a common cell. No mutations were detected in the PDGFRA, KRAS, BRAF, $N F 1$, and TP53 genes. SDHA mutations were identified only in the high-grade tumor of case 4.

Thirty genes mutated only in high-grade GISTs were detected in 247 oncogenes. Mutations in five genes were detected in two cases (MGA, ARIDIA, CLTC, KAT6B, and ZNF521), while the others were mutated in only one patient. We detected an average of 3.5 differential cancer-associated genes per patient with a range of 1-6 genes. Twenty four oncogenes mutated only in low-grade tumors were detected, and six genes were examined in no less than two cases.

The differential cancer-associated gene mutations were verified by Sanger sequencing. Ten differential mutations found only in high-grade tumors were confirmed in nine genes: MGA, SDHA, ARID1A, LATS2, MAX, SETD2, RB1, RPS6KB2, and PIK3CA (Table 2 and Fig. 4), among which the first seven are tumor suppressor genes, and the latter two are oncogenes. These mutations were present in seven of ten patients. The mutational profile did not show any highly recurrent differential alterations shared by the majority of cases, and only $M G A$ variants were identified in two

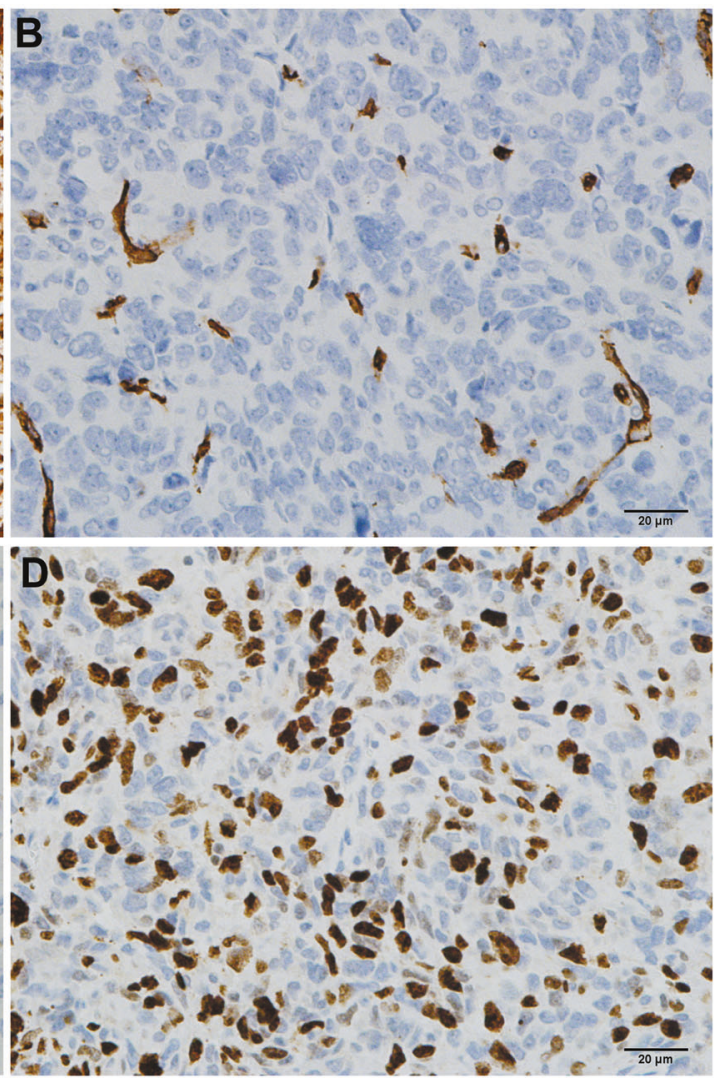

CD34 negative expression (IHC, $\times 400$ ). The Ki-67 proliferation index in the low-grade tumor was $\sim 3 \%$ (c), while in high-grade tumors, the Ki-67 proliferation index was $\sim 30 \%$ (d) (IHC, $\times 400$ )

patients, while four patients had concurrent differential mutations. Functional enrichment analysis highlighted many significantly enriched pathways related to cell proliferation (MGA, MAX, RB1, ARIDIA, PIK3CA, RPS6KB1, LATS2), evasion of apoptosis (PIK3CA, RPS6KB1, SDHA, LATS2), and chromatin remodeling (ARIDIA and SETD2).

$M G A$ mutations were identified in high-grade tumors from two cases of small intestinal GISTs: a stop-gain mutation (c.4741C>T, p.Q1581*) and a frameshift deletion (c.1599delT, p.H533fs*12). Both mutations were predicted to generate a premature stop codon and to cause truncation of the C-terminal region of the MGA protein, including the basic helix-loop-helix leucine zipper (bHLHLZ) domain. The patient with the MGA stop-gain mutation also had additional differential alterations in $S D H A$, which appeared as two consecutive base substitutions (c.363G $>$ A, p.W121* and c.364C $>\mathrm{T}$, p.H122Y), and resulted in a significant reduction in SDHA protein expression (Fig. 5) (Anti-SDHA antibody [EPR9043(B)], Abcam, epitope was a synthetic peptide within Human SDHA C-terminal 550-650aa). In addition to mutations in $M G A$ and $S D H A$, stop-gain mutations were identified in the ARIDIA (c.666C >A, p.Y222*) and $R B 1$ (c.496G $>$ T, p.E166*) genes, which may lead to the 

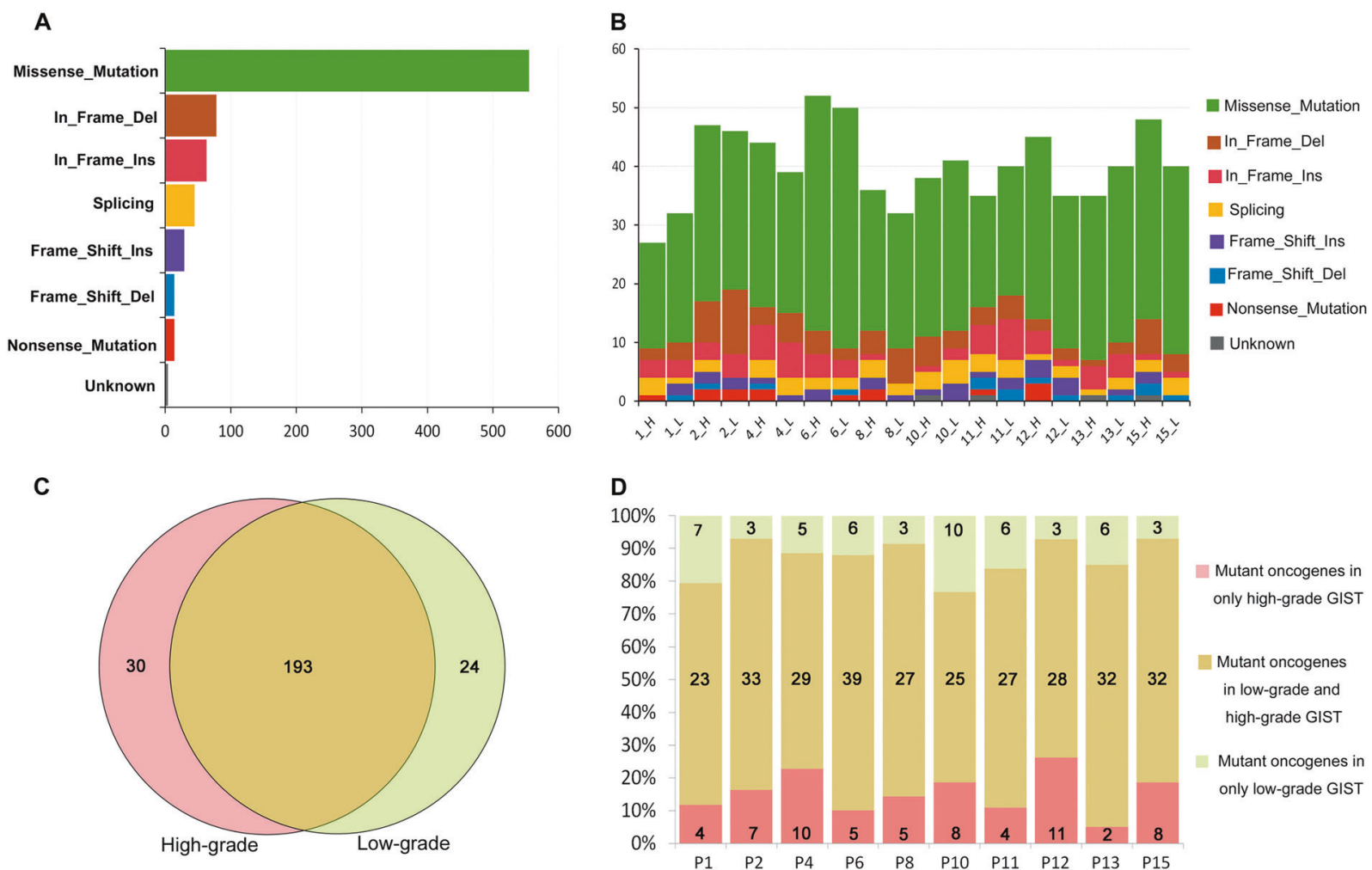

Fig. 3 The mutation features of cancer-associated genes identified by WES. a WES in ten cases of high-grade GIST and matched low-grade GIST showed variant mutation patterns with a predominance of missense mutations. b Every specimen exhibited different mutation patterns, and the number of mutations in each tumor ranged from 27 to 52. c Venn diagram analysis of mutational genes in all high- and lowgrade GISTs revealed that both components share many similar

loss of function of these tumor suppressor genes. The tumor with an ARIDIA mutation also had a missense mutation in SETD2 (c.6074T >C, p.I2025T). A PIK3CA missense SNV (c.333G $>$ T, p.K111N) was confirmed in one case of gastric GIST. The frequency of PIK3CA mutations in GIST is $\sim 1.65 \%$ (14/846), while this is the first report of the PIK3CA mutation c.333G $>\mathrm{T}$ in GIST. A MAX somatic frameshift insertion (c.216_217insAC, p.Q73fs) in the bHLHLZ domain was detected in a female patient. This mutation may lead to amino acid changes after codon 73 , which were predicted to result in the loss of MAX protein function. For the first time, missense SNVs in LATS2 (c.2144C $>\mathrm{G}, \quad$ p.A715G) and RPS6KB2 (c.1261C $>\mathrm{A}$, p.P421T) were identified in high-grade GIST components.

\section{MYC copy number gain may promote tumor progression of GIST}

Copy-number analysis based on WES data for MYC genes showed that three high-grade tumors carried copy-number gains compared with their corresponding low-grade tumors. One case had MYC amplification validated by FISH, which

mutations in cancer-associated genes (numbers represent the number of genes). d An analysis of the proportion of mutated cancerassociated genes in high- and low-grade GISTs from each patient showed that most mutated genes were detected in both components and that a small number of differential genes were mutated only in high-grade or low-grade tumors (numbers in histograms represent the number of genes)

illustrated four red/two green signals in the high-grade tumor and two red/two green signals in the low-grade tumor (Fig. 6), while the remaining two cases showed chromosome 8 polysomy with copy number gain in the $M Y C$ gene in both high-grade and low-grade tumors.

MYC CNV was assessed in 22 pairs of GIST with coexisting low- and high-grade components by FISH. In low-grade tumors, 15 cases had chromosome 8 polysomy with copy number gain of the $M Y C$ gene, and 7 cases showed normal $M Y C$ gene copy number; in contrast, in high-grade tumors, 17 cases demonstrated chromosome 8 polysomy with copy number gain, one case had MYC amplification, and four cases showed normal MYC gene copy number (Table 3). High-grade GISTs had more $M Y C$ gene copy number gains than low-grade GISTs $(P=0.012)$, but no significant differences were found in the number of cases of MYC copy number gain between high-grade tumors and low-grade tumors $(P=0.296$, Table 3$)$. It was suggested that the alterations in $M Y C$ copy number occurred in the early stages of GIST (low-grade GIST) and that more $M Y C$ copy number gains contributed to GIST progression. 


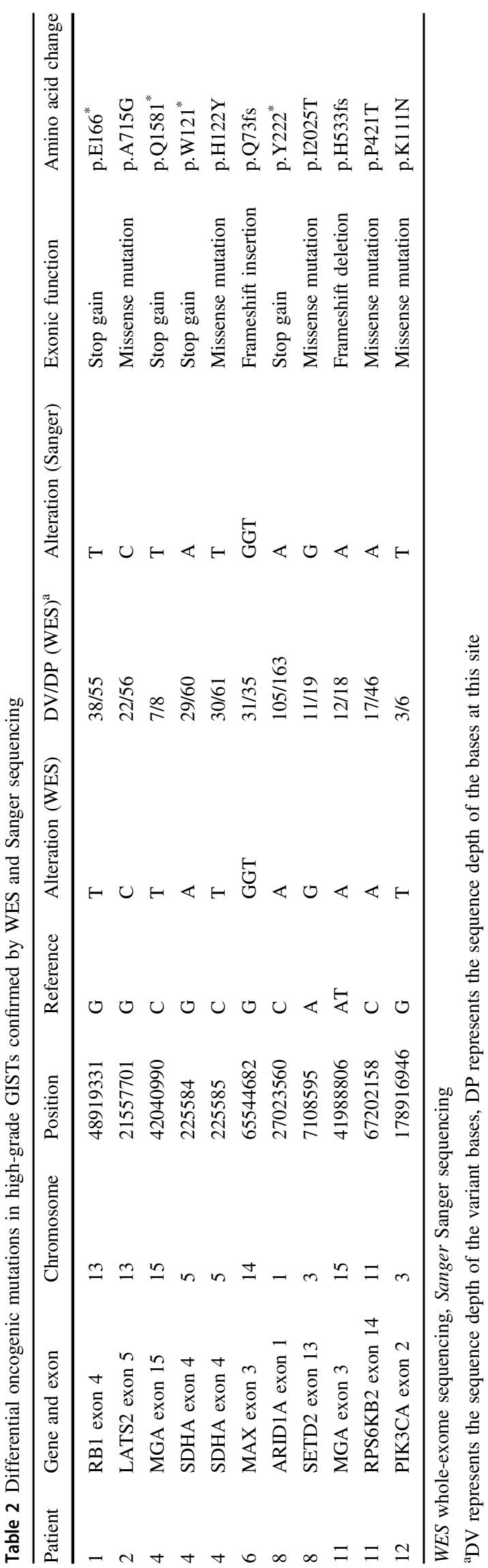

Detection of 86 cases of pure low- or high-grade GIST by FISH showed similar results. In 60 cases of low-grade GIST, 43 had normal MYC copy number, and 17 had chromosome 8 polysomy with copy number gain of the $M Y C$ gene; in 26 cases of high-grade GIST, normal MYC copy number was detected in ten cases, chromosome 8 polysomy with copy number gain of the $M Y C$ gene was detected in 15 cases, and $M Y C$ amplification was detected in one case (Table 3). MYC CNV in high-grade GIST had more copy number gains compared with low-grade GIST $(P=0.004$, Table 3$)$. Moreover, the tumors with MYC copy number gain usually had a higher mitotic index and a higher Ki-67 proliferation index $(P=0.010$ and 0.001 , respectively, Table 3). Compared with pure low-grade GISTs, low-grade tumors with coexisting high-grade components had a higher incidence of $M Y C$ copy number gain $(P=$ 0.001), which suggested that low-grade GISTs with $M Y C$ copy number gain were more likely to transform into highgrade tumors and that $M Y C$ copy number gain might promote tumor progression.

\section{Discussion}

Despite harboring similar driver KIT mutations, most GISTs exhibit extremely different clinical behaviors, which vary widely from incidental, clinically silent small tumors to locally invasive, distant metastatic tumors with high mitotic activity [12]. Additional genetic events may influence the variable behavior of these tumors, and highgrade tumors are likely to harbor many genomic alterations associated with highly aggressive behavior and high proliferative activity [13-15]. In the present study, WES results revealed 30 and 24 oncogenes that mutated only in high-grade GISTs and low-grade tumors, respectively. These mutations might occur during tumor progression, and the mutations only in low-grade tumors did not cause GIST progression to high-grade tumor. Tumor subclones with mutations only in low-grade tumors may have different subclonal origins from high-grade tumors. Furthermore, we identified ten differential genetic alterations in nine genes in seven patients by Sanger sequencing, and seven of these genes (MGA, MAX, RB1, ARIDIA, PIK3CA, RPS6KB1, LATS2) have been documented to be involved in cell cycle regulation. Therefore, we deduced that mutations in these genes were associated with tumor progression of low-grade to high-grade tumors and that the progression of GISTs may be the result of cell cycle control via the convergent integration of various cascades, such as the MGA/MAX/MYC network and the PI3K/AKT signaling pathway (Fig. S2). Notably, none of the patients received chemotherapy or receptor tyrosine kinase inhibitor therapy prior to surgery, which indicates that the 


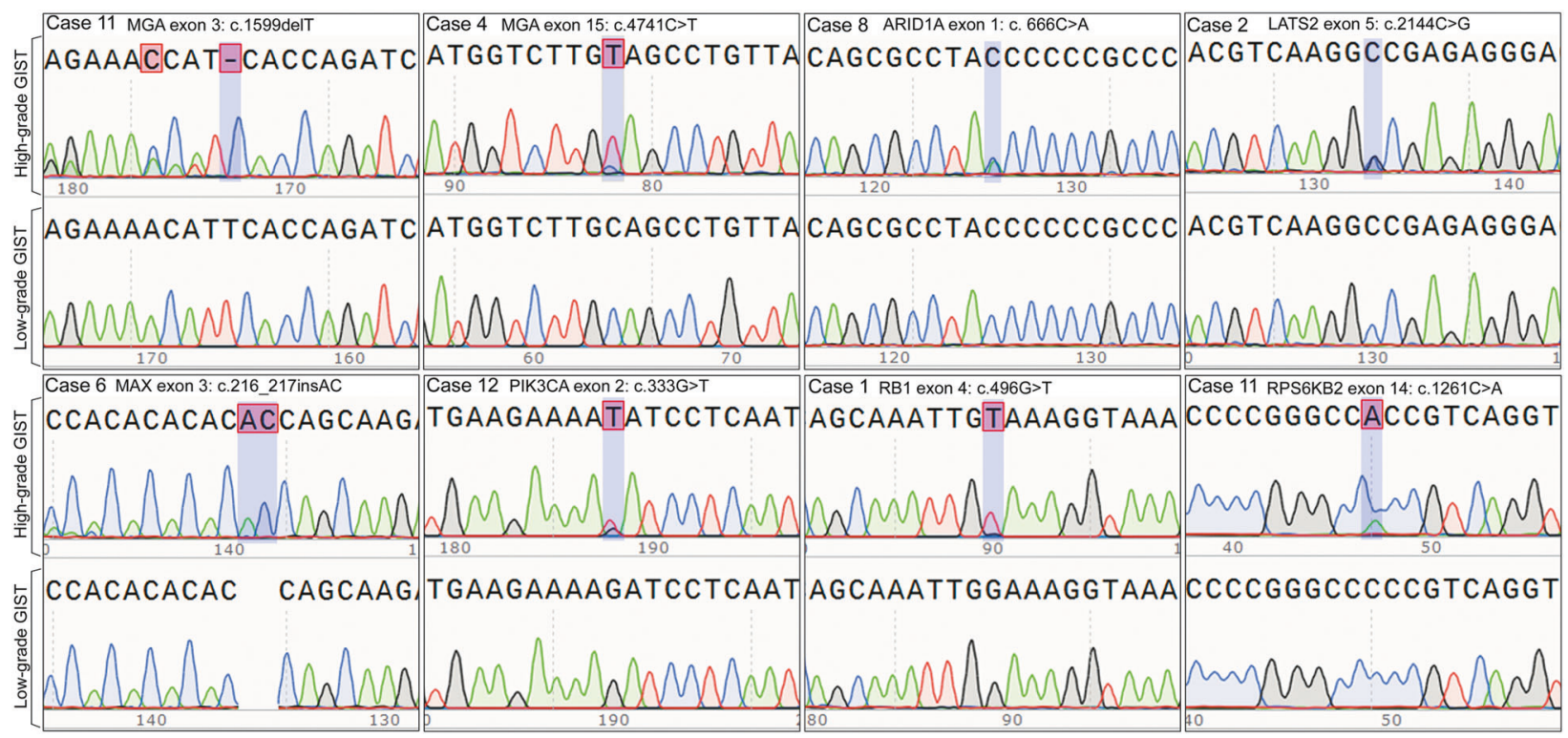

Fig. 4 Representative differential oncogenic mutations identified in high-grade GISTs by Sanger sequencing. In each image, the top panel depicts the sequencing fluorescent peak trace chromatograms of high- grade GIST, and the bottom panel depicts the low-grade tumor counterpart (mutations are highlighted by light blue squares) mutations identified in our study were spontaneous events unrelated to any therapy.

MGA and MAX are members of the bHLHLZ family of transcription factors. In our study, truncating mutations in $M G A$ were identified only in the high-grade tumors of two cases of small bowel GIST. In addition, $M G A$ mutations (c.5554C > T, p.H1852Y and c.7766delT, p.I2589fs) have been reported in two other cases of small bowel GIST with KIT mutations [11]. All reported MGA mutations in GISTs occurred in high-grade tumors or metastatic tumors, which suggest that MGA mutations might promote tumor progression. A possible mechanism for this is that $M G A$ lossof-function mutations may reduce the suppression of MAX-MYC heterodimerization complex formation and promote GIST progression by regulating MYC-dependent transformation $[16,17]$, which modulates the cell cycle by enhancing cyclin-dependent kinase (CDK) functions. Furthermore, we identified a $M A X$ loss-of-function mutation in the present study. $M A X$ mutations are not considered a rare genetic event in GIST, and MAX inactivation may foster cell cycle activity by regulating $\mathrm{p} 16^{\mathrm{INK} 4 \mathrm{~A}}$ silencing [18].

PIK3CA is a downstream effector of the KIT/PDGFRA signaling pathway that drives carcinogenesis by inducing proliferation and inhibiting apoptosis [19, 20]. In the present study, a PIK3CA mutation was detected only in the high-grade component in a KIT-mutant GIST of a patient who had not undergone targeted therapy with tyrosine kinase inhibitors, which suggests that PIK3CA mutations may represent primary oncogenic events related to GIST progression. Although the $P I K 3 C A$ c. $333 \mathrm{G}>\mathrm{T}$ mutation was first reported in GIST, it occurs in a variety of tumors (18 reported cases in COSMIC: mutation ID COSM27505), which suggests that this mutation is a recurrent genetic event that is a vital contributor to oncogenesis and progression. GISTs with PIK3CA base substitution mutations are frequently classified as high-risk tumors [21], and more $P I K 3 C A$ mutations are detected in large and metastatic KITmutant GISTs, which suggests that PIK3CA mutant clones have a proliferative advantage during disease progression [4, 21]. Moreover, oncogenic activation of the PI3K pathway has been implicated in different cancers and contributes to a more aggressive tumor phenotype $[22,23]$. In addition, the loss of ARID1A has recently been linked to increased AKT phosphorylation and PI3K/AKT pathway activation [24]. In the present study, a stop-gain mutation in ARIDIA in a high-grade GIST was validated in one patient with extensive abdominal metastasis. PIK3CA and ARIDIA mutations can activate the PI3K/AKT pathway and further activate CDK which is complexed with cyclins, and leads to tumor cell proliferation and inhibition of apoptosis [25].

$R B 1$ is one of the most important genes in cell cycle regulation. In our study, one case had an $R B 1$ loss-offunction mutation only in high-grade GIST. Similarly, Merten et al. identified two cases of $R B 1$ inactivating missense mutations in GIST, which were correlated with morphologically high-risk features and clinically malignant behavior [26]. Moreover, deletion of the RBI gene locus identified by fluorescence in situ hybridization is frequent in high-risk GIST and may be associated with tumor progression [27]. To our knowledge, RPS6KB2 and LATS2 mutations in GIST have not been previously reported. RPS6KB2 is an important kinase regulated by the mTOR signaling 

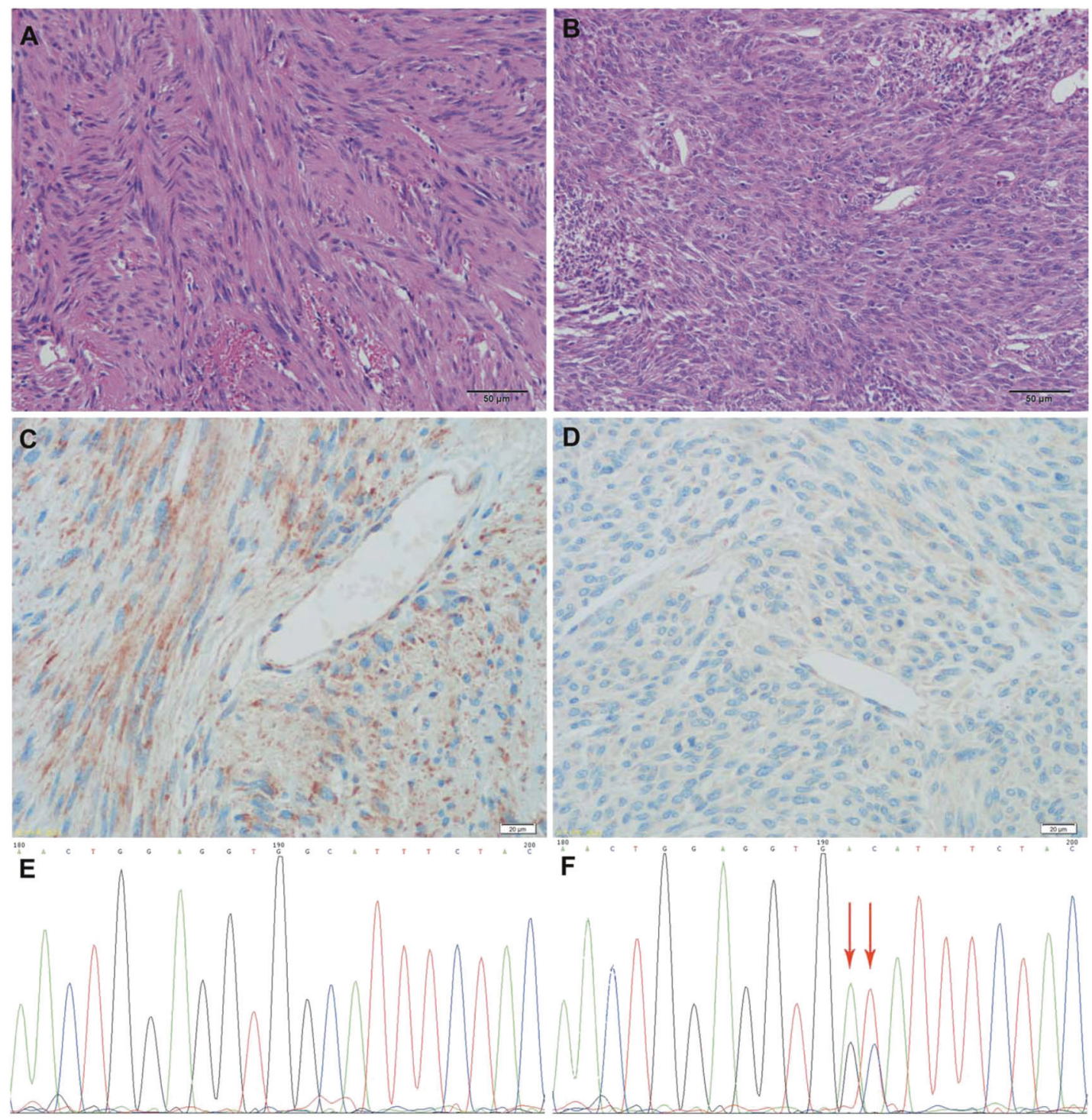

Fig. 5 SDHA mutations resulted in decreased expression of SDHA protein. a Morphological characteristics of low-grade GIST components in case 4 (HE, ×200); b Morphological features of high-grade tumors (HE, $\times 200)$. c Immunohistochemical results of low-grade tumors revealed moderate to strong cytoplasmic expression of SDHA

pathway, and its activity can shorten the G1 phase of the cell cycle, enabling cells to enter $\mathrm{S}$ phase at an increased rate by binding several heterogenous ribonuclear proteins in a mitogen-inducible manner [28]. Since RPS6KB2 is an oncogene, activating mutations may fuel tumor progression by regulating cell cycle control. LATS2 is a component of the Hippo signaling pathway, which is involved in regulating cell proliferation by inhibiting CDK2/cyclin E activity and limiting G1/S transition [29, 30]. In addition, LATS2 can promote DREAM (DP, RB, E2F, and MuvB) repressor complex assembly by phosphorylating DYRK1A and can promote RB-induced permanent cell cycle arrest in tumor cells [31]. In the present study, a LATS2 mutation was found protein $(\mathrm{IHC}, \times 400)$; d High-grade tumors showed a marked reduction in SDHA expression (IHC, $\times 400$ ). e Sanger sequencing revealed wildtype SDHA in low-grade GIST; f SDHA mutations (c.363_364GC>AT) in high-grade tumors were detected by Sanger sequencing

in the protein kinase-like domain, which may undermine the tumor-suppressor function of this gene.

MYC is a positive regulator of G1-specific CDKs and acts at least three distinct pathways: (1) functional inactivation of the CDK inhibitors $\mathrm{p} 27^{\mathrm{Kip} 1}$, p $21^{\mathrm{Cip} 1}$, and $\mathrm{p} 57^{\mathrm{Kip} 2}$, (2) induction of the CDK-activating phosphatase Cdc25A and (3) deregulation of cyclin E expression [32]. MYC gene alterations are common genetic variations, as $21 \%$ of solid tumors show MYC copy number gain [17]. In this study, $50.8 \%$ of GIST samples showed MYC copy number gain, especially GISTs with coexisting low- and high-grade components. Low-grade GIST with copy number gain led to a higher frequency of tumor progression, and the tumors with $M Y C$ copy number 

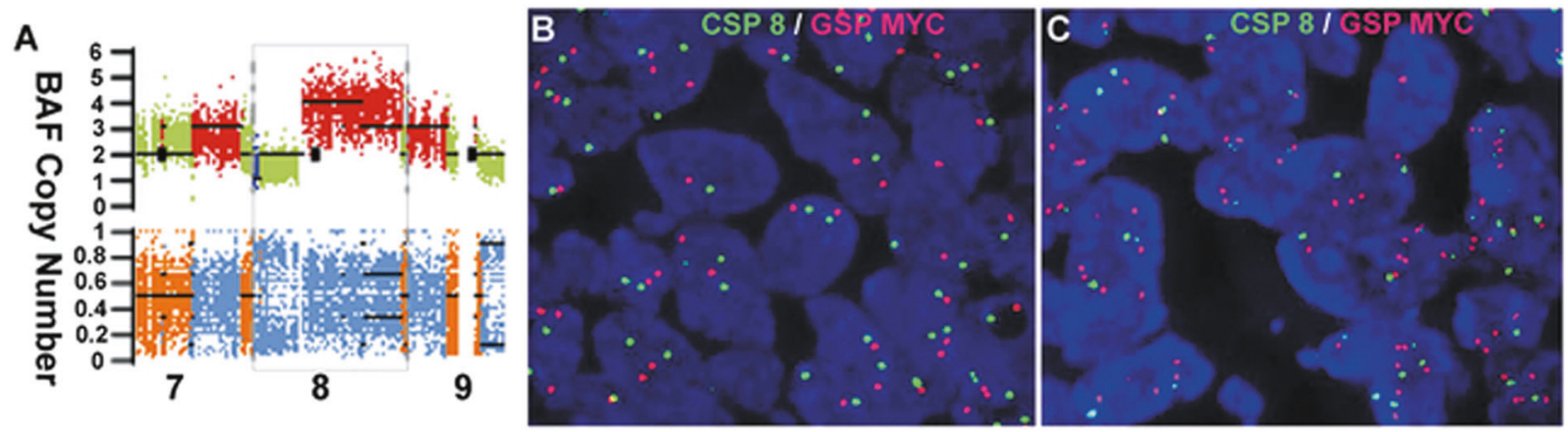

Fig. 6 MYC CNV in low-grade GIST and high-grade GIST of case 11. a CNV analysis revealed an increased copy number of the chromosome 8 fragment (inside the square frame) where $M Y C$ resides (the upper figure: red represents an increase in copy number, blue represents a decrease in copy number, and green indicates no change in copy number; the lower figure: orange indicates the same allele distribution, while blue indicates the preference of allele distribution); b Normal $M Y C$ copy number (two red/two green signals) was detected in the low-grade tumor; c $M Y C$ copy number amplification (four red/ two green signals) was identified in the high-grade tumor
Table 3 The relationship among $M Y C$ copy number gain and tumor grade, mitotic index and Ki-67 proliferation index

\begin{tabular}{|c|c|c|c|c|c|c|}
\hline \multirow[t]{2}{*}{ Tumor features } & \multicolumn{3}{|c|}{ Coexisting GIST } & \multicolumn{3}{|l|}{ Pure GIST } \\
\hline & $\begin{array}{l}M Y C \text { copy } \\
\text { number gain }\end{array}$ & $\begin{array}{l}M Y C \text { copy } \\
\text { number normal }\end{array}$ & $P$ & $\begin{array}{l}M Y C \text { copy } \\
\text { number gain }\end{array}$ & $\begin{array}{l}M Y C \text { copy } \\
\text { number normal }\end{array}$ & $P$ \\
\hline \multicolumn{3}{|l|}{ Tumor grade } & 0.296 & & & 0.004 \\
\hline Low-grade & 15 & 7 & & 17 & 43 & \\
\hline High-grade & 18 & 4 & & 16 & 10 & \\
\hline \multicolumn{3}{|c|}{ Mitotic index (/50 HPF) } & 0.174 & & & 0.010 \\
\hline$\leq 5$ & 15 & 7 & & 17 & 43 & \\
\hline $6-10$ & 7 & 4 & & 4 & 7 & \\
\hline $11-20$ & 3 & 0 & & 2 & 0 & \\
\hline$>20$ & 8 & 0 & & 10 & 3 & \\
\hline \multicolumn{3}{|c|}{ Ki-67 proliferation index $(\%)$} & 0.153 & & & 0.001 \\
\hline$\leq 5$ & 11 & 6 & & 10 & 27 & \\
\hline $6-10$ & 3 & 1 & & 8 & 19 & \\
\hline $11-30$ & 14 & 4 & & 8 & 6 & \\
\hline$>30$ & 5 & 0 & & 7 & 1 & \\
\hline
\end{tabular}

gain usually had a higher mitotic index and a higher Ki-67 proliferation index. These findings suggest that MYC copy number gain may promote progression of GISTs by regulating tumor cell proliferative activity.

In summary, the present study reports 22 GIST cases with concomitant low- and high-grade components, both of which displayed different histopathological and immunohistochemical characteristics. WES was performed using ten pairs of high-grade GISTs and matched low-grade GISTs, and the data showed that both components shared most of the detected mutations in cancer-associated genes. WES and Sanger sequencing identified nine cancerassociated genes that were mutated only in high-grade GISTs; the function of these genes is primarily cell cycle control. FISH revealed that MYC copy number gain is a common genetic alteration in GIST and that this alteration may occur in the early stages of this disease. It was also revealed that low-grade tumors with $M Y C$ copy number gain were more likely to progress to high-grade tumors. GISTs with $M Y C$ copy number gain had higher proliferative activity. Therefore, the primary progression of GISTs may be the result of a variety of different genes acting through different signaling pathways, and cell cycle regulationrelated gene mutations and copy number gain of the $M Y C$ gene may play important roles in these pathways.

Funding The study was funded by grants from the National Natural Science Foundation of China (81570180 to ZW, 81570176 to QY), and the State Key Laboratory of Cancer Biology (CBSKL201725 to PL).

\section{Compliance with ethical standards}

Conflict of interest For the remaining authors none were declared.

Publisher's note Springer Nature remains neutral with regard to jurisdictional claims in published maps and institutional affiliations. 


\section{References}

1. Rubin BP, Heinrich MC, Corless CL. Gastrointestinal stromal tumour. Lancet. 2007;369:1731-41.

2. Boikos SA, Pappo AS, Killian JK, LaQuaglia MP, Weldon CB, George S, et al. Molecular subtypes of KIT/PDGFRA wild-type gastrointestinal stromal tumors: a report from the national institutes of health gastrointestinal stromal tumor clinic. JAMA. Oncol. 2016;2:922-8.

3. Gill AJ. Succinate dehydrogenase (SDH)-deficient neoplasia. Histopathology. 2018;72:106-16.

4. Lasota J, Felisiak-Golabek A, Wasag B, Kowalik A, Zieba S, Chlopek M, et al. Frequency and clinicopathologic profile of PIK3CA mutant GISTs: molecular genetic study of 529 cases. Mod Pathol. 2016;29:275-82.

5. Pantaleo MA, Urbini M, Indio V, Ravegnini G, Nannini M, De Luca M, et al. Genome-wide analysis identifies MEN1 and MAX mutations and a neuroendocrine-like molecular heterogeneity in quadruple WT GIST. Mol Cancer Res. 2017;15:553-62.

6. Brenca M, Rossi S, Polano M, Gasparotto D, Zanatta L, Racanelli D, et al. Transcriptome sequencing identifies ETV6-NTRK3 as a gene fusion involved in GIST. J Pathol. 2016;238:543-9.

7. Huang KK, McPherson JR, Tay ST, Das K, Tan IB, Ng CC, et al. SETD2 histone modifier loss in aggressive GI stromal tumours. Gut. 2016;65:1960-72.

8. Vogelstein B, Papadopoulos N, Velculescu VE, Zhou S, Diaz LA Jr, Kinzler KW. Cancer genome landscapes. Science. 2013;339: 1546-58.

9. Kandoth C, McLellan MD, Vandin F, Ye K, Niu B, Lu C, et al. Mutational landscape and significance across 12 major cancer types. Nature. 2013;502:333-9.

10. Tamborero D, Gonzalez-Perez A, Perez-Llamas C, Deu-Pons J, Kandoth C, Reimand J, et al. Comprehensive identification of mutational cancer driver genes across 12 tumor types. Sci Rep. 2013;3:2650.

11. Zehir A, Benayed R, Shah RH, Syed A, Middha S, Kim HR, et al. Mutational landscape of metastatic cancer revealed from prospective clinical sequencing of 10,000 patients. Nat Med. 2017;23:703-13.

12. Joensuu H, Hohenberger P, Corless CL. Gastrointestinal stromal tumour. Lancet. 2013;382:973-83.

13. Okamoto Y, Sawaki A, Ito S, Nishida T, Takahashi T, Toyota M, et al. Aberrant DNA methylation associated with aggressiveness of gastrointestinal stromal tumour. Gut. 2012;61:392-401.

14. Schneider-Stock R, Boltze C, Lasota J, Peters B, Corless CL, Ruemmele $\mathrm{P}$, et al. Loss of p16 protein defines high-risk patients with gastrointestinal stromal tumors: a tissue microarray study. Clin Cancer Res. 2005;11:638-45.

15. Ihle MA, Huss S, Jeske W, Hartmann W, Merkelbach-Bruse S, Schildhaus HU, et al. Expression of cell cycle regulators and frequency of TP53 mutations in high risk gastrointestinal stromal tumors prior to adjuvant imatinib treatment. PLoS One. 2018;13: e0193048.

16. Carroll PA, Freie BW, Mathsyaraja H, Eisenman RN. The MYC transcription factor network: balancing metabolism, proliferation and oncogenesis. Front Med. 2018;12:412-25.
17. Schaub FX, Dhankani V, Berger AC, Trivedi M, Richardson AB, Shaw R, et al. Pan-cancer alterations of the MYC oncogene and its proximal network across the cancer genome atlas. Cell Syst. 2018;6:282-300 e2.

18. Schaefer IM, Wang Y, Liang CW, Bahri N, Quattrone A, Doyle L, et al. MAX inactivation is an early event in GIST development that regulates $\mathrm{p} 16$ and cell proliferation. Nat Commun. 2017;8:14674.

19. Long ZW, Wu JH, Cai H, Wang YN, Zhou Y. MiR-374b promotes proliferation and inhibits apoptosis of human GIST cells by inhibiting PTEN through activation of the PI3K/Akt pathway. Mol Cells. 2018;41:532-44.

20. Vivanco I, Sawyers CL. The phosphatidylinositol 3-Kinase AKT pathway in human cancer. Nat Rev Cancer. 2002;2:489-501.

21. Daniels M, Lurkin I, Pauli R, Erbstosser E, Hildebrandt U, Hellwig K, et al. Spectrum of KIT/PDGFRA/BRAF mutations and Phosphatidylinositol-3-Kinase pathway gene alterations in gastrointestinal stromal tumors (GIST). Cancer Lett. 2011;312: $43-54$.

22. Ogino S, Nosho K, Kirkner GJ, Shima K, Irahara N, Kure S, et al. PIK3CA mutation is associated with poor prognosis among patients with curatively resected colon cancer. J Clin Oncol. 2009;27:1477-84.

23. Samuels Y, Diaz LA Jr, Schmidt-Kittler O, Cummins JM, Delong L, Cheong I, et al. Mutant PIK3CA promotes cell growth and invasion of human cancer cells. Cancer Cell. 2005;7:561-73.

24. Samartzis EP, Gutsche K, Dedes KJ, Fink D, Stucki M, Imesch P. Loss of ARID1A expression sensitizes cancer cells to PI3K- and AKT-inhibition. Oncotarget. 2014;5:5295-303.

25. Gao N, Flynn DC, Zhang Z, Zhong XS, Walker V, Liu KJ, et al. G1 cell cycle progression and the expression of G1 cyclins are regulated by $\mathrm{PI} 3 \mathrm{~K} / \mathrm{AKT} / \mathrm{mTOR} / \mathrm{p} 70 \mathrm{~S} 6 \mathrm{~K} 1$ signaling in human ovarian cancer cells. Am J Physiol Cell Physiol. 2004;287: C281-91.

26. Merten L, Agaimy A, Moskalev EA, Giedl J, Kayser C, Geddert $\mathrm{H}$, et al. Inactivating mutations of RB1 and TP53 correlate with sarcomatous histomorphology and metastasis/recurrence in gastrointestinal stromal tumors. Am J Clin Pathol. 2016; 146:718-26.

27. Zhou W, Zeng X, Liu T. Aberrations of chromosome $13 \mathrm{q}$ in gastrointestinal stromal tumors: analysis of 91 cases by fluorescence in situ hybridization (FISH). Diagn Mol Pathol. 2009;18:72-80.

28. Pardo OE, Seck1 MJ. S6K2: the neglected S6 kinase family member. Front Oncol. 2013;3:191.

29. Furth N, Aylon Y. The LATS1 and LATS2 tumor suppressors: beyond the Hippo pathway. Cell Death Differ. 2017;24:1488-501.

30. Tao W, Zhang S, Turenchalk GS, Stewart RA, St John MA, Chen $\mathrm{W}$, et al. Human homologue of the Drosophila melanogaster lats tumour suppressor modulates $\mathrm{CDC} 2$ activity. Nat Genet. 1999;21:177-81.

31. Tschop K, Conery AR, Litovchick L, Decaprio JA, Settleman J, Harlow E, et al. A kinase shRNA screen links LATS2 and the pRB tumor suppressor. Genes Dev. 2011;25:814-30.

32. Amati B, Alevizopoulos K, Vlach J. Myc and the cell cycle. Front Biosci. 1998;3:d250-68. 